\title{
ZnO Nanosheets Decorated with Graphite-Like Carbon Nitride Quantum Dots as Photoanodes in Photoelectrochemical Water Splitting
}

\author{
Chavi Mahala ${ }^{1}$, Mamta Devi Sharma ${ }^{1}$, Mrinmoyee Basu ${ }^{1 *}$ \\ ${ }^{1}$ Department of Chemistry, BITS Pilani, Pilani Campus, Rajasthan-333031 \\ mrinmoyee.basu@gmail.com
}

Chemicals: Trisodium citrate dehydrate purchased from SRL. Urea, Zinc nitrate hexahydrate $\left(\mathrm{Zn}\left(\mathrm{NO}_{3}\right)_{2} \cdot 6 \mathrm{H}_{2} \mathrm{O}\right)$, and Potassium chloride $(\mathrm{KCl})$ were purchased from SDFCL. Researchgrade solvent ethanol was purchased from Spectrochem. Dialysis sack (molecular cutoff weight $(\mathrm{MW})=10000 \mathrm{kDa}$ ) was purchased from Sigma-Aldrich. All chemicals were used as purchased without any further purification.

Characterizations: Shimadzu Spectrophotometer (model no. UV-2450) with a deuterium and tungsten-halogen lamp was used to study ultra-violate visible spectroscopy. Horiba Jobin Yvon Spectroflourimeter (Fluoro max-4) was used to measure the photoluminescence (PL) spectra. Powder X-ray analysis was carried out using Rigaku Mini Flex II diffractometer with incident radiation of $\mathrm{Cu}-\mathrm{K} \alpha$. Whole analysis was carried out keeping the scanning rate $2^{\circ}$ per min. Microscope version, XT Platform version, XT UI version, Modal- "APREO S" FE-SEM was used to investigate the morphology of the synthesized $\mathrm{ZnO} 2 \mathrm{D}$ nanosheets by $\mathrm{C}_{3} \mathrm{~N}_{4}$ QDs. EDS Analysis was carried out for these samples using the EDS attachment with FESEM which is Aztec (software), X-MaxN, NS: 77887 (Detector) of Oxford company. Raman analysis was carried out using HORIBASCI Raman instrument (model no LabRAM HR EVO). The detector is thermoelectrically cooled charged coupled device (CCD) detector of $576 \times 384$ pixels. Raman analysis is carried out upon excitation with $532 \mathrm{~nm}$ laser power. XPS (X-ray photoelectron spectroscopy) computation was carried out by Omicron EA 125 source using Al Ka radiation having energy $1486.7 \mathrm{eV}$. During the course of measurement, base pressure was maintained $<10^{-9} \mathrm{mbar}$ in the UHV.

Photoelectrochemical Measurement: In this present case, photoelectrochemical study was conducted in a three-electrode system. PEC water splitting was carried out $0.5 \mathrm{M} \mathrm{Na}_{2} \mathrm{SO}_{4}$ as the electrolyte. In the cell silver chloride electrode was applied as reference electrode, $\mathrm{Pt}$ as the counter electrode and sample deposited FTO as working electrode. Xenon lamp was used to illuminate the PEC cell with fixed light intensity of $100 \mathrm{~mW} / \mathrm{cm}^{2}$. $\mathrm{CH}$ Instrument (CHI604E) was used to record all the electrochemical data at $25^{\circ} \mathrm{C}$. Initially, linear-sweep voltammogram (LSV) was recorded for $\mathrm{ZnO}$ and $\mathrm{ZnO} / \mathrm{C}_{3} \mathrm{~N}_{4}$-QDs photoanode upon applied potential from $-0.9 \mathrm{~V}$ to $1 \mathrm{~V}$ vs. $\mathrm{Ag} / \mathrm{AgCl}$ keeping scan rate $20 \mathrm{mV} / \mathrm{sec}$ under back illumination condition. Chronoamperometry study was carried out under potential of $0.0 \mathrm{~V}$ vs. $\mathrm{Ag} / \mathrm{AgCl}$.

Electrochemical Impedance Study: Electrochemical impedance measurement was also performed in a three electrode system. It was performed at a bias of $0.0 \mathrm{~V}$ vs $\mathrm{Ag} / \mathrm{AgCl}$ 
electrode with the sweeping of frequency from $50 \mathrm{kHZ}$ to $1 \mathrm{~Hz}$. Xenon lamp was used to illuminate the PEC cell with fixed light intensity of $100 \mathrm{~mW} / \mathrm{cm}^{2}$.
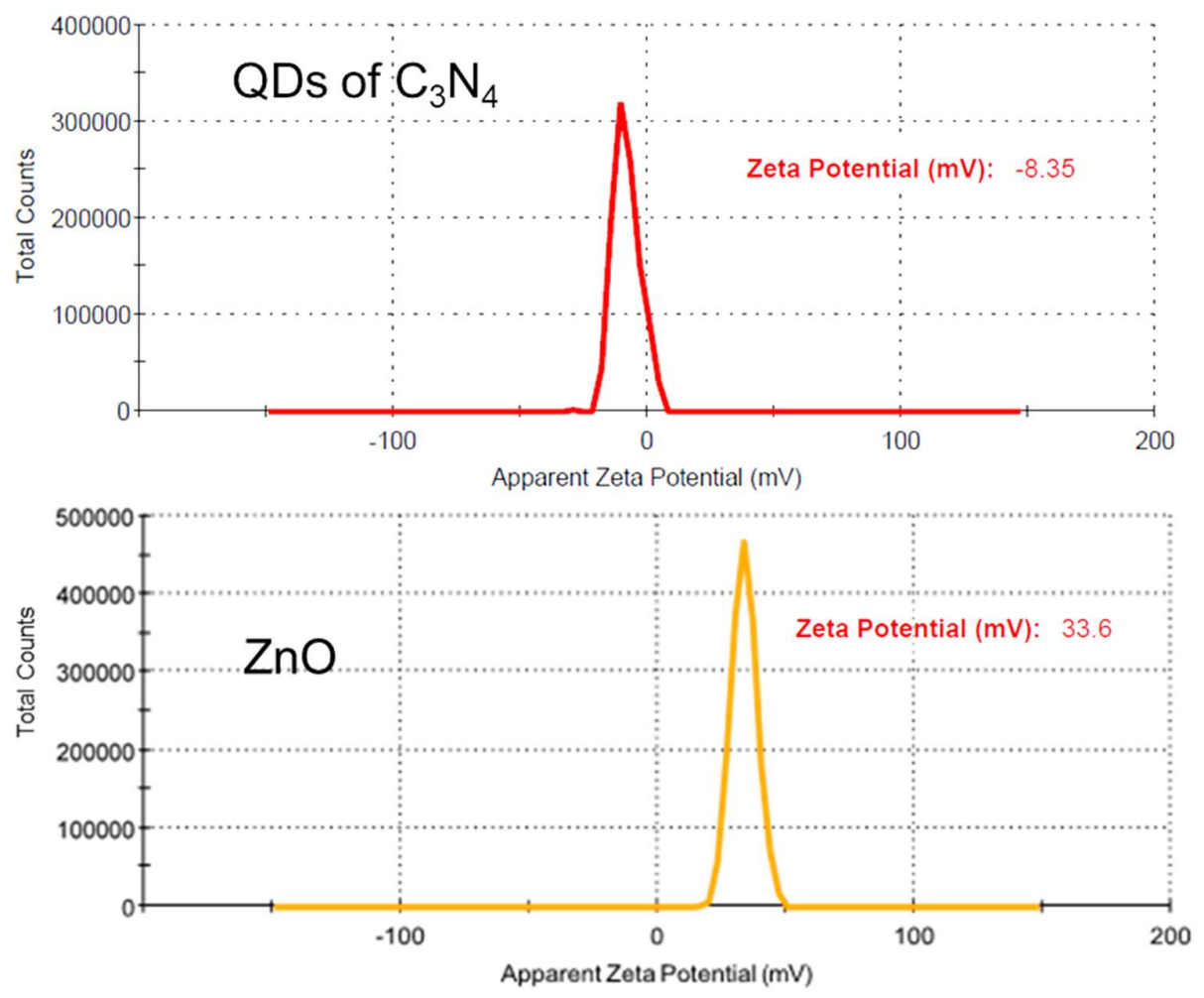

Figure S1: Zeta potential measurement data of $\mathrm{C}_{3} \mathrm{~N}_{4}$ QDs and $\mathrm{ZnO}$. 

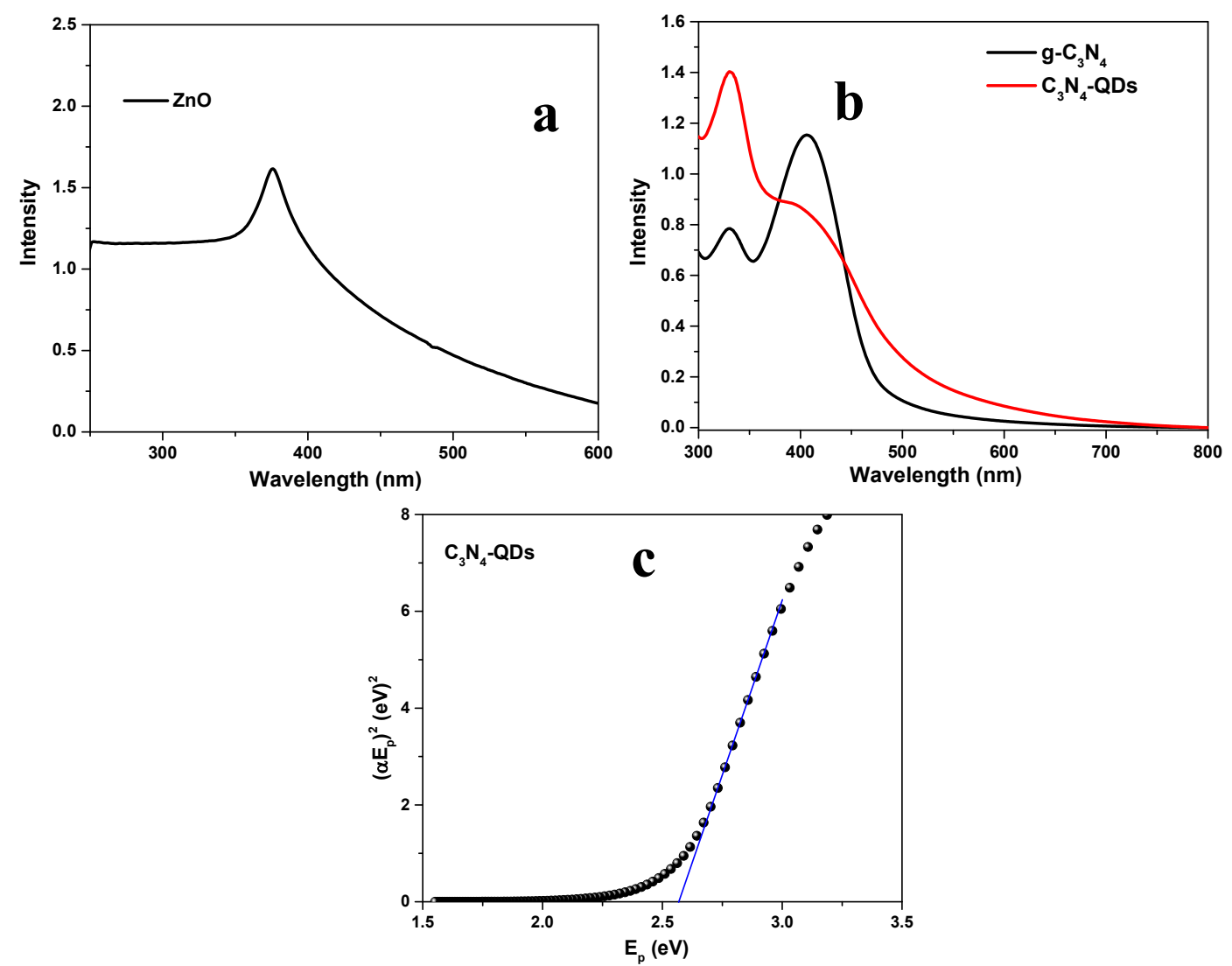

Figure S2: UV-vis spectra of (a) $\mathrm{ZnO}$ nanosheets (b) $\mathrm{g}-\mathrm{C}_{3} \mathrm{~N}_{4}$, and $\mathrm{C}_{3} \mathrm{~N}_{4}-\mathrm{QDs}$, (c) Band gap calculation of $\mathrm{C}_{3} \mathrm{~N}_{4}-\mathrm{QDs}$. 


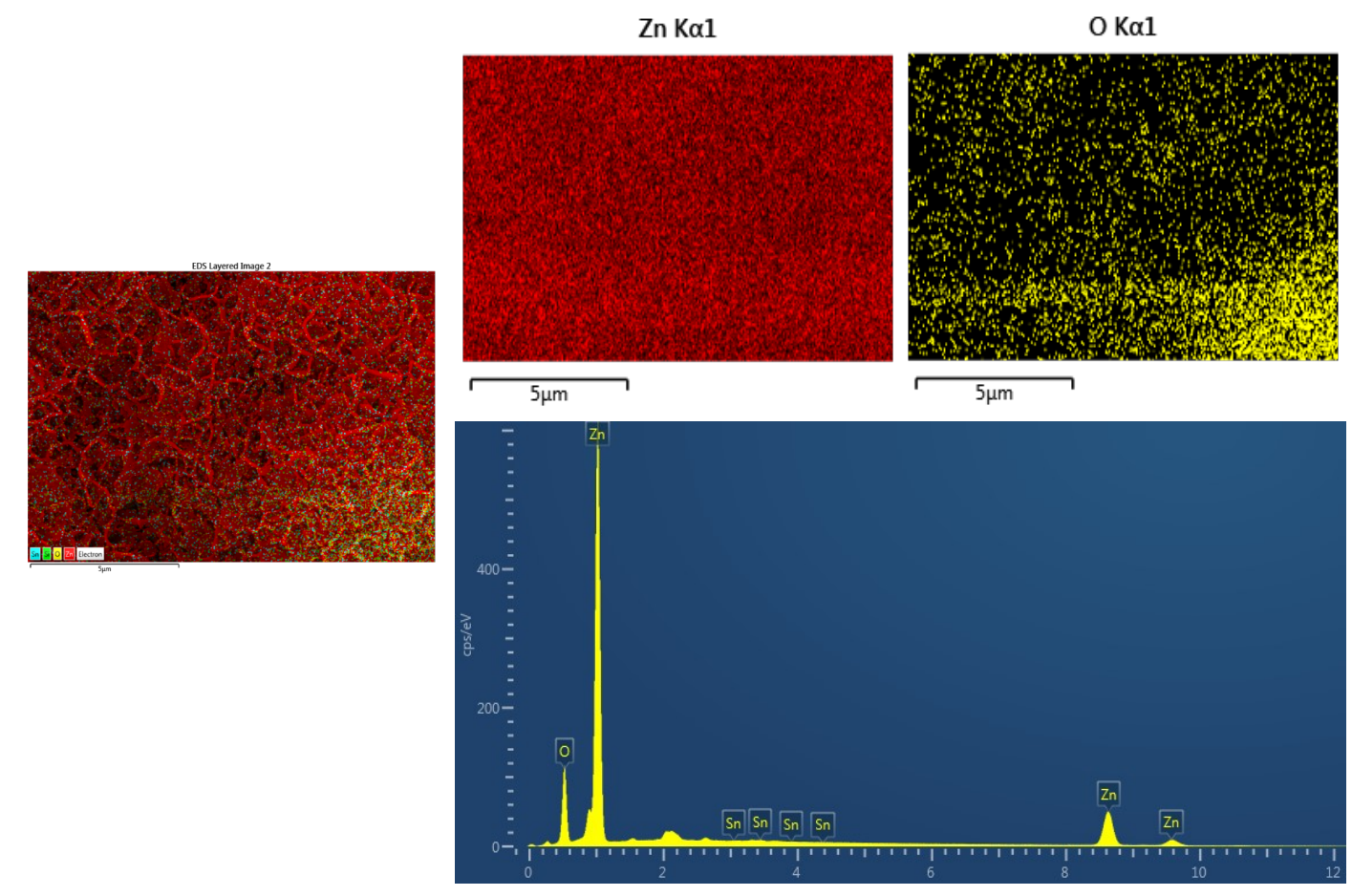

Figure S3: EDS analysis of $\mathrm{ZnO}$ nanosheets on FTO which shows the uniform distribution of 'Zn', 'O'. 


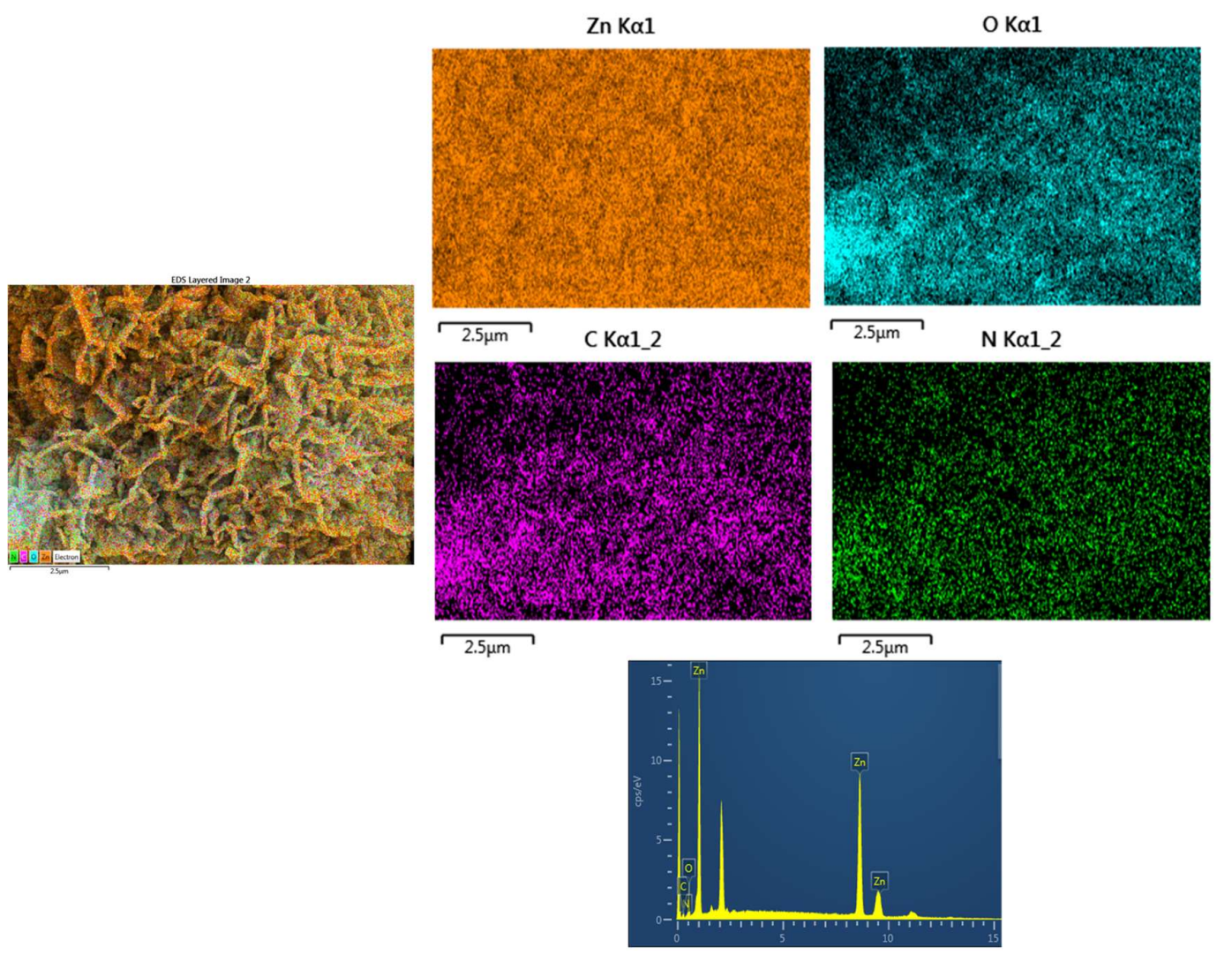

Figure S4: EDS analysis of $\mathrm{ZnO} / \mathrm{C}_{3} \mathrm{~N}_{4}-15$ nanosheets on $\mathrm{FTO}$ which shows the uniform distribution of ' $\mathrm{Zn}$ ', ' $\mathrm{O}$ ', ' $\mathrm{C}$ ' and ' $\mathrm{N}$ ' 


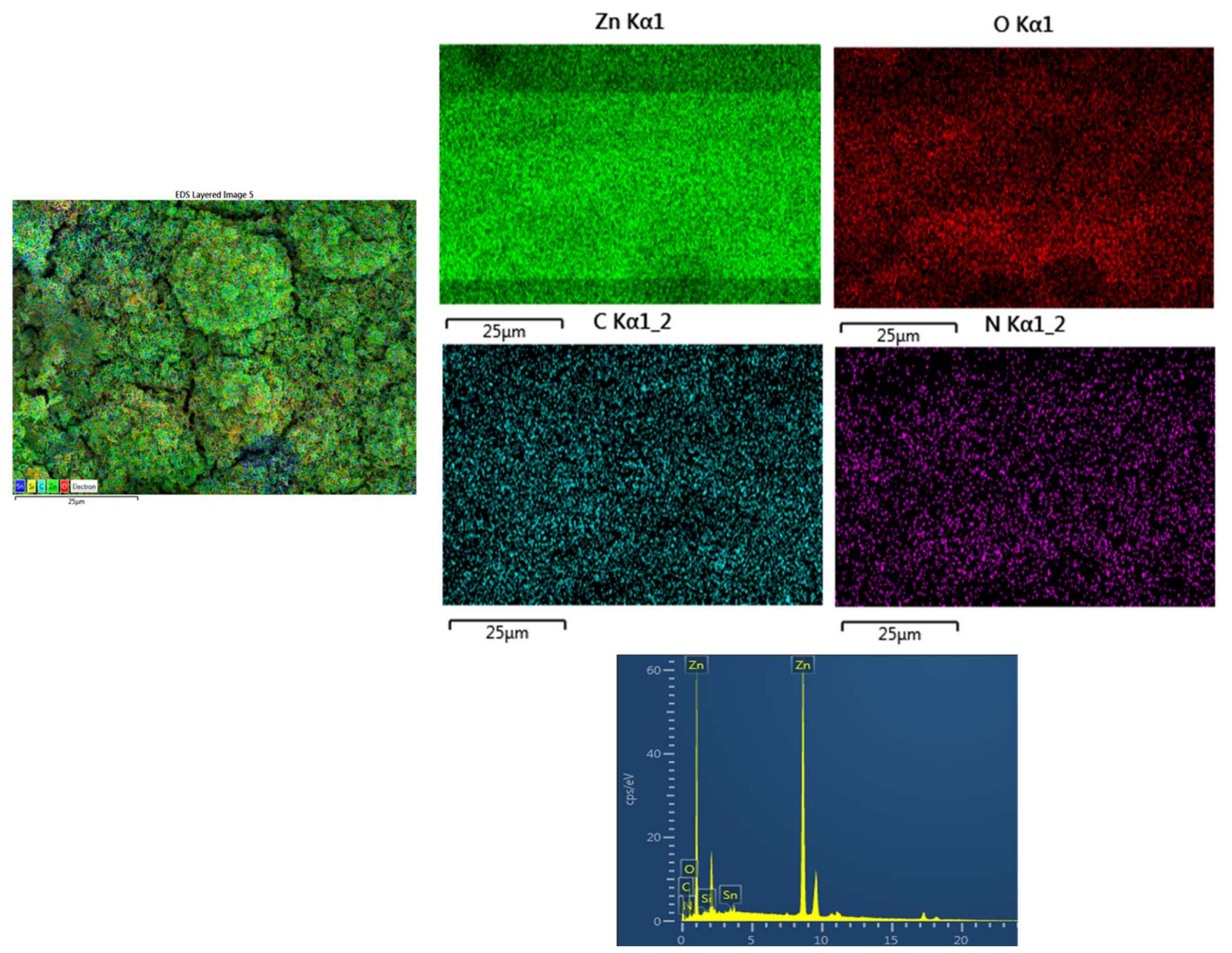

Figure S5: EDS analysis of $\mathrm{ZnO} / \mathrm{C}_{3} \mathrm{~N}_{4}-30$ on FTO which shows the uniform distribution of 'Zn', 'O', 'C' and 'N'. 

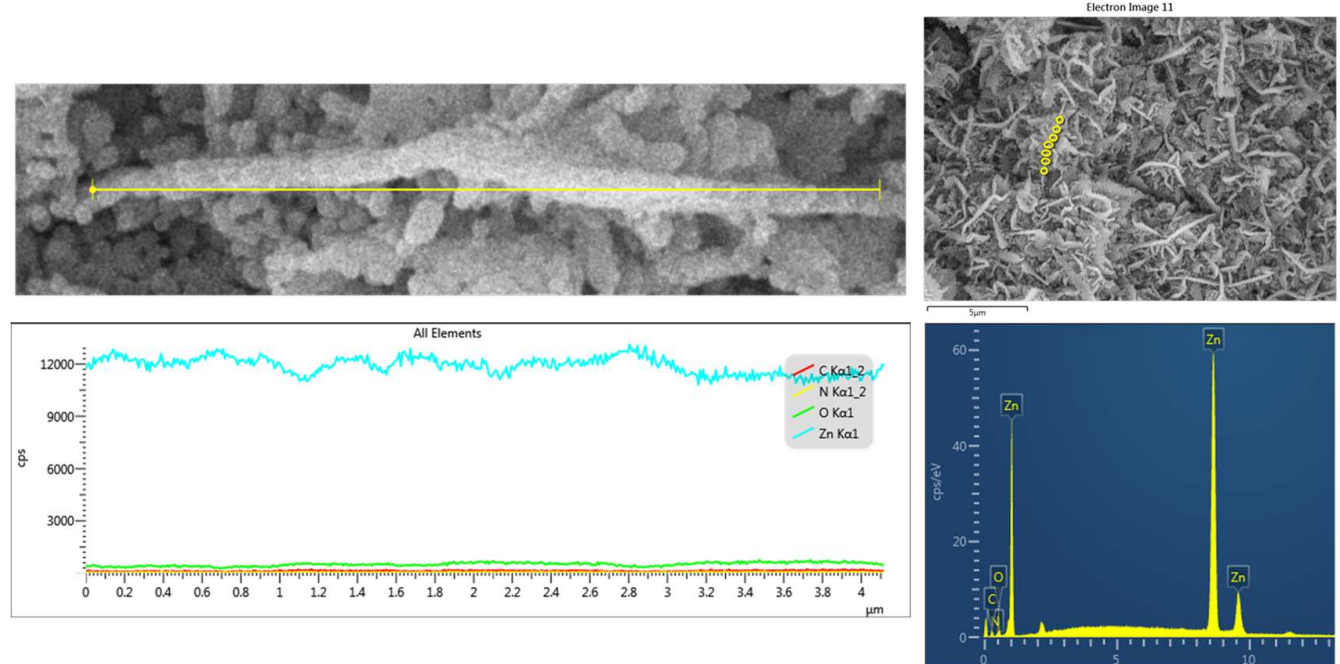

Figure S6: Line mapping in FESEM-EDS analysis of $\mathrm{ZnO} / \mathrm{C}_{3} \mathrm{~N}_{4}-15$, which shows the relative presence of ' $\mathrm{Zn}$ ', 'O', 'C' and ' $\mathrm{N}$ ' elements.
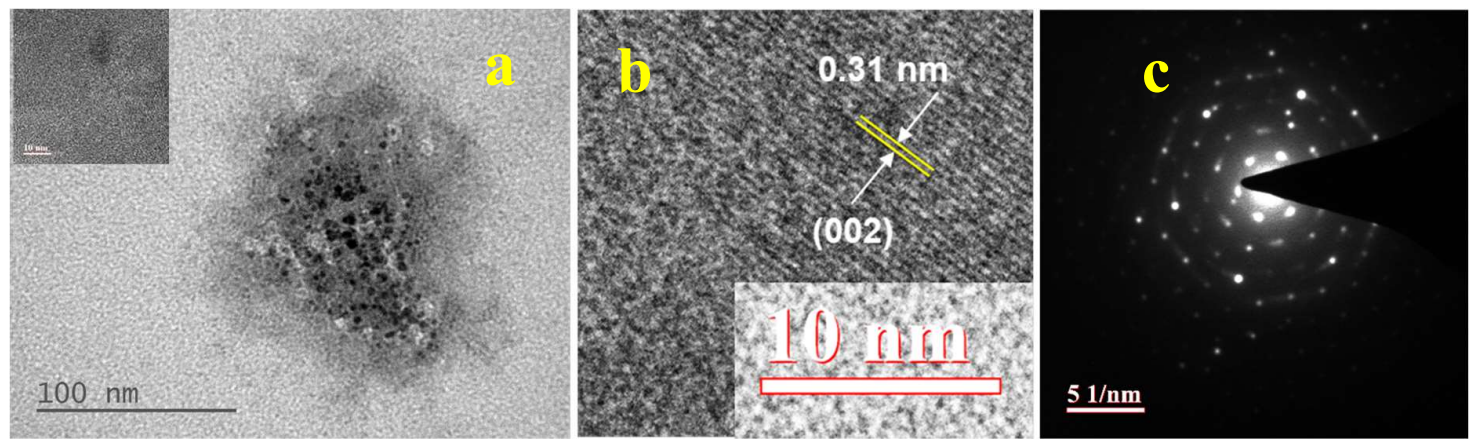

Figure S7: (a) TEM image, (b) HRTEM image, (c) SAED pattern of $\mathrm{C}_{3} \mathrm{~N}_{4}$ QDs. 

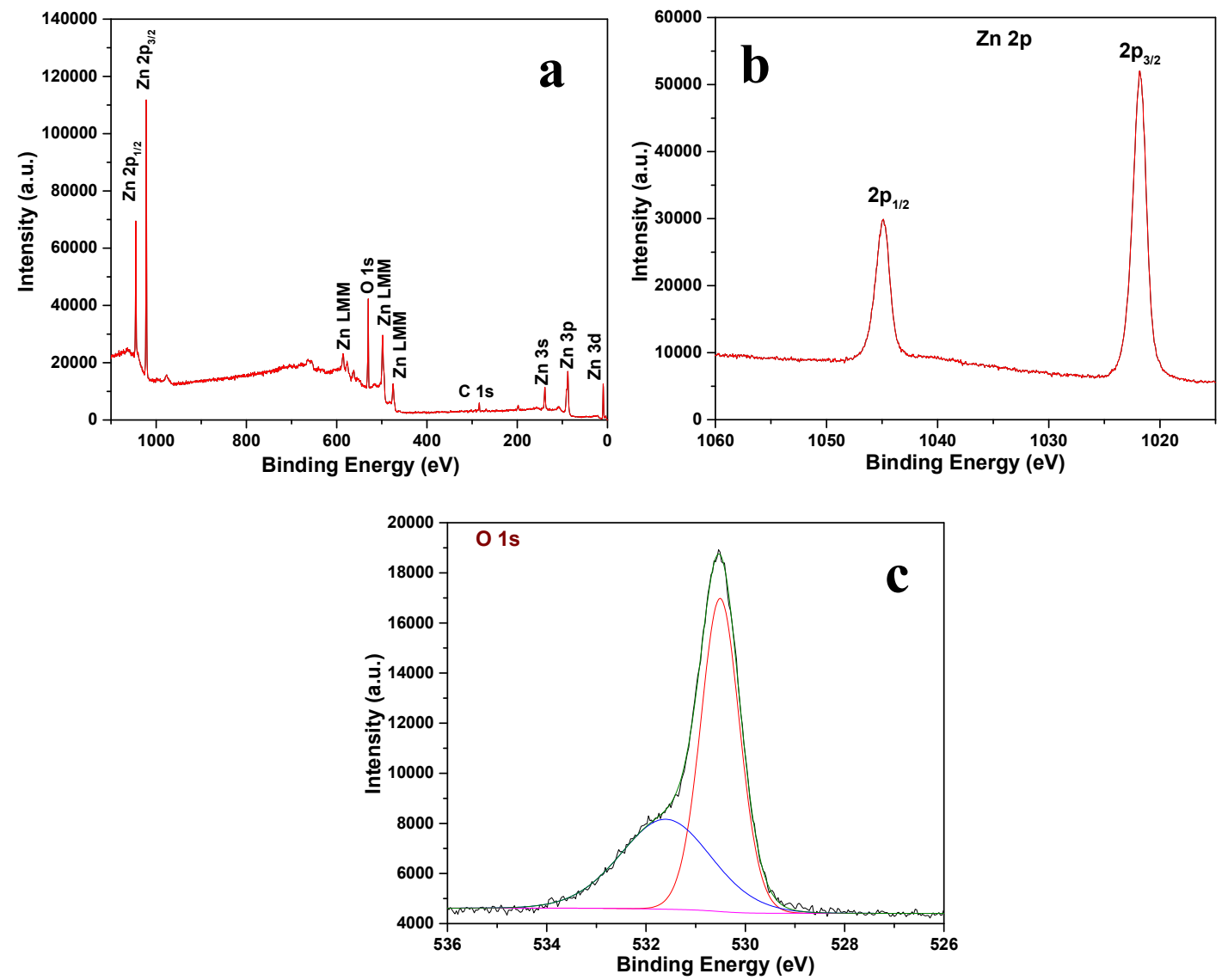

Figure S8: XPS spectra of bare ZnO (a) survey spectra, (b) Zn 2p, (c) O1s. 


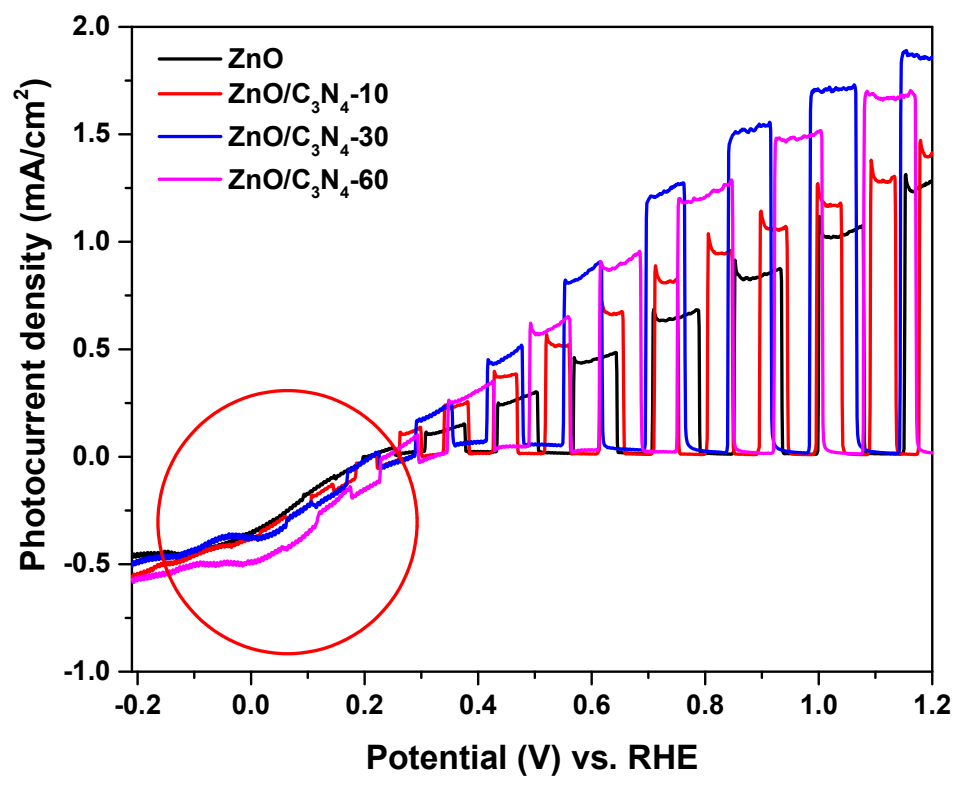

Figure S9: Linear sweep voltammograms of $\mathrm{ZnO}, \mathrm{ZnO} / \mathrm{C}_{3} \mathrm{~N}_{4}-10,30$, and 60 under chopped illumination condition. 

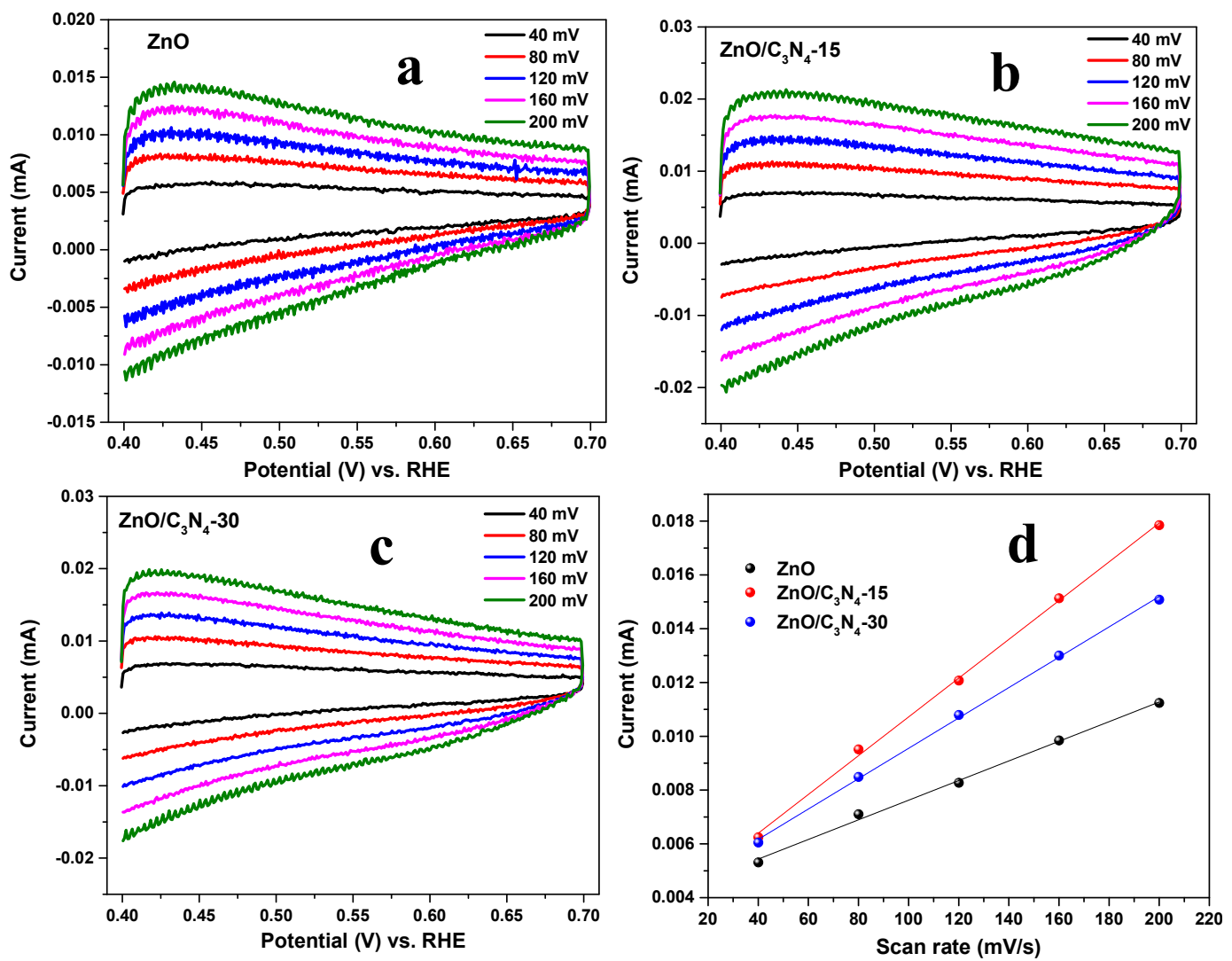

Figure S10: $a, b$, and c represents the cyclic voltammograms curves and (d) represents electrochemical double-layer capacitance of $\mathrm{ZnO}, \mathrm{ZnO} / \mathrm{C}_{3} \mathrm{~N}_{4}-15$ and $\mathrm{ZnO} / \mathrm{C}_{3} \mathrm{~N}_{4}-30$ respectively. 
Table S1: Comparative PEC performance data of $\mathrm{ZnO} / \mathrm{C}_{3} \mathrm{~N}_{4}-\mathrm{QD}$ heterostructures at different potentials.

\begin{tabular}{|c|c|c|}
\hline Photoanodes & $\begin{array}{c}\text { Photocurrent density } \\
\left(\mathrm{mA} / \mathrm{cm}^{2}\right) \\
\text { at } \\
\text { RHE } 0.5994^{+} \mathrm{V} \text { vs. }\end{array}$ & $\begin{array}{c}\text { Photocurrent density } \\
\left(\mathrm{mA} / \mathrm{cm}^{2}\right) \\
\text { V vs. RHE }\end{array}$ \\
\hline $\mathrm{ZnO}$ & 0.414 & 1.32 \\
\hline $\mathrm{ZnO} / \mathrm{C}_{3} \mathrm{~N}_{4}-10$ & 0.647 & 1.68 \\
\hline $\mathrm{ZnO} / \mathrm{C}_{3} \mathrm{~N}_{4}-15$ & 0.952 & 2.26 \\
\hline $\mathrm{ZnO} / \mathrm{C}_{3} \mathrm{~N}_{4}-30$ & 0.852 & 2.06 \\
\hline $\mathrm{ZnO} / \mathrm{C}_{3} \mathrm{~N}_{4}-60$ & 0.704 & 1.95 \\
\hline
\end{tabular}


Table S2: Comparative PEC performance data of $\mathrm{ZnO} / \mathrm{C}_{3} \mathrm{~N}_{4}-\mathrm{QDs}$ heterostructure with the reported data in literature.

\begin{tabular}{|c|c|c|c|}
\hline Ref. & Photoelectrode & Photocurrent density & Elelctrolyte \\
\hline \multirow[t]{3}{*}{ S1 } & $\begin{array}{l}\text { Ternary } \\
\text { g- } \mathrm{C}_{3} \mathrm{~N}_{4} / \mathrm{Pt} / \mathrm{ZnO}\end{array}$ & $120 \mu \mathrm{A} / \mathrm{cm}^{2}$ at $0.5 \mathrm{~V}$ vs. $\mathrm{Ag} / \mathrm{AgCl}$ & \multirow[t]{3}{*}{$\begin{array}{l}0.5 \mathrm{M} \\
\mathrm{Na}_{2} \mathrm{SO}_{4}\end{array}$} \\
\hline & $\mathrm{g}-\mathrm{C}_{3} \mathrm{~N}_{4} / \mathrm{ZnO}$ & $26.7 \mu \mathrm{A} / \mathrm{cm}^{2}$ at $0.5 \mathrm{~V}$ vs. $\mathrm{Ag} / \mathrm{AgCl}$ & \\
\hline & $\mathrm{ZnO}$ & $13 \mu \mathrm{A} / \mathrm{cm}^{2}$ at $0.5 \mathrm{~V}$ vs. $\mathrm{Ag} / \mathrm{AgCl}$ & \\
\hline \multirow[t]{3}{*}{ S2 } & $\begin{array}{l}\mathrm{ZnO} / \mathrm{Au} / \mathrm{g}-\mathrm{C}_{3} \mathrm{~N}_{4} \\
\text { photocathode }\end{array}$ & $-0.91 \mathrm{~mA} / \mathrm{cm}^{2}$ at $-0.4 \mathrm{~V}$ vs. RHE & \multirow[t]{3}{*}{$\begin{array}{l}0.2 \mathrm{M} \\
\mathrm{Na}_{2} \mathrm{SO}_{4}\end{array}$} \\
\hline & $\mathrm{ZnO} / \mathrm{g}-\mathrm{C}_{3} \mathrm{~N}_{4}$ & $-0.49 \mathrm{~mA} / \mathrm{cm}^{2}$ at $-0.4 \mathrm{~V}$ vs. RHE & \\
\hline & & $-0.13 \mathrm{~mA} \mathrm{~cm}^{-2}$ at $-0.4 \mathrm{~V}$ vs. RHE & \\
\hline \multirow[t]{2}{*}{ S3 } & $\begin{array}{l}\mathrm{g}_{-} \mathrm{C}_{3} \mathrm{~N}_{4} / \mathrm{TiO}_{2} \text { Nanotube } \\
\text { Array }\end{array}$ & $0.86 \mathrm{~mA} / \mathrm{cm}^{2}$ at $0.7 \mathrm{~V}$ vs. $\mathrm{Ag} / \mathrm{AgCl}$ & \multirow[t]{2}{*}{$\begin{array}{l}0.1 \mathrm{M} \\
\mathrm{Na}_{2} \mathrm{SO}_{4}\end{array}$} \\
\hline & $\mathrm{TiO}_{2}$ & $0.07 \mathrm{~mA} / \mathrm{cm}^{2}$ at $0.7 \mathrm{~V}$ vs. $\mathrm{Ag} / \mathrm{AgCl}$ & \\
\hline \multirow[t]{2}{*}{38} & $\begin{array}{l}\text { CNQDs/ZnO } \\
\text { composites }\end{array}$ & $3.8 \mathrm{~mA} / \mathrm{cm}^{2}$ at $0.6 \mathrm{~V}$ vs. $\mathrm{SCE}$ & \multirow[t]{2}{*}{$\begin{array}{l}0.2 \mathrm{M} \\
\mathrm{Na}_{2} \mathrm{SO}_{4}\end{array}$} \\
\hline & $\mathrm{ZnO}$ nanowire arrays & $0.8 \mathrm{~mA} / \mathrm{cm}^{2}$ at $0.6 \mathrm{~V}$ vs. $\mathrm{SCE}$ & \\
\hline \multirow[t]{2}{*}{ S4 } & $\mathrm{CdS} / \mathrm{g}-\mathrm{C}_{3} \mathrm{~N}_{4} / \mathrm{ZnO}$ & $3.34 \mathrm{~mA} / \mathrm{cm}^{2}$ at $1.23 \mathrm{~V}$ vs. RHE & \multirow{2}{*}{$\begin{array}{l}0.1 \mathrm{M} \mathrm{Na} \mathrm{Na}_{2} \mathrm{~S} \\
\text { and } 0.2 \quad \mathrm{M} \\
\mathrm{Na}_{2} \mathrm{SO}_{3} \\
(\mathrm{~V}: \mathrm{V}=1: 1)\end{array}$} \\
\hline & $\mathrm{g}-\mathrm{C}_{3} \mathrm{~N}_{4} / \mathrm{ZnO}$ & $0.7 \mathrm{~mA} / \mathrm{cm}^{2}$ at $1.23 \mathrm{~V}$ vs. RHE & \\
\hline \multirow[t]{2}{*}{ S5 } & $\mathrm{ZnO} / \mathrm{g}-\mathrm{C}_{\mathrm{x}} \mathrm{N}_{\mathrm{y}}(1.0)$ & $0.25 \mathrm{~mA} / \mathrm{cm}^{2}$ at $1.23 \mathrm{~V}$ versus RHE & \multirow[t]{2}{*}{$0.1 \mathrm{M} \mathrm{KOH}$} \\
\hline & $\mathrm{ZnO}$ & $0.075 \mathrm{~mA} / \mathrm{cm}^{2}$ at $1.23 \mathrm{~V}$ versus RHE & \\
\hline S6 & $\begin{array}{l}\text { Y:ZnO@PCN core- } \\
\text { shell NR arrays }\end{array}$ & $0.4 \mathrm{~mA} / \mathrm{cm}^{2}$ at $1.23 \mathrm{~V}$ vs RHE & $\begin{array}{l}0.5 \mathrm{M} \\
\mathrm{Na}_{2} \mathrm{SO}_{4}\end{array}$ \\
\hline \multirow[t]{2}{*}{36} & $\mathrm{TiO}_{2} @ \mathrm{~g}-\mathrm{CNQDNWs}$ & $3.40 \mathrm{~mA} / \mathrm{cm}^{2}$ at ' 0 ' $\mathrm{V}$ at vs $\mathrm{Ag} / \mathrm{AgCl}$ & \multirow[t]{2}{*}{$1 \mathrm{M} \mathrm{KOH}$} \\
\hline & $\mathrm{TiO}_{2} \mathrm{NW}$ & $1.36 \mathrm{~mA} / \mathrm{cm}^{2}$ at ' 0 ' $\mathrm{V}$ at vs $\mathrm{Ag} / \mathrm{AgCl}$ & \\
\hline \multirow[t]{2}{*}{ S7 } & g- $\mathrm{C}_{3} \mathrm{~N}_{4} / \mathrm{TiO}_{2}$ nanotubes & $125.32 \mu \mathrm{A} / \mathrm{cm}^{2}$ at '0' V vs. SCE & \multirow{2}{*}{$\begin{array}{l}0.5 \mathrm{M} \\
\mathrm{Na}_{2} \mathrm{SO}_{4}\end{array}$} \\
\hline & $\mathrm{TiO}_{2}$ nanotubes & $72.54 \mu \mathrm{A} / \mathrm{cm}^{2}$ at ' 0 ' $\mathrm{V}$ vs. SCE & \\
\hline \multirow{2}{*}{$\begin{array}{l}\text { This } \\
\text { work }\end{array}$} & $\mathrm{ZnO} 2 \mathrm{D}$ sheets & $0.414 \mathrm{~mA} / \mathrm{cm}^{2}$ at $0.5994 \mathrm{~V}$ vs. RHE & \multirow{2}{*}{$\begin{array}{l}0.5 \mathrm{M} \\
\mathrm{Na}_{2} \mathrm{SO}_{4}\end{array}$} \\
\hline & $\begin{array}{l}\mathrm{ZnO} / \mathrm{C}_{3} \mathrm{~N}_{4} \\
\text { heterostructure }\end{array}$ & $0.952 \mathrm{~mA} / \mathrm{cm}$ at $0.5994 \mathrm{~V}$ vs. RHE & \\
\hline
\end{tabular}




\section{References:}

S1. Xiao, J.; Zhang, X.; Li, Y., A ternary $\mathrm{g}-\mathrm{C}_{3} \mathrm{~N}_{4} / \mathrm{Pt} / \mathrm{ZnO}$ photoanode for efficient photoelectrochemical water splitting. Int. J. Hydrog. Energy, 2015, 40, 9080-9087.

S2. Wen, P.; Sun, Y.; Li, H.; Liang, Z.; Wu, H.; Zhang, J.; Zeng, H.; Geyer, S. M.; Jiang, L. A highly active three-dimensional $\mathrm{Z}$-scheme $\mathrm{ZnO} / \mathrm{Au} / \mathrm{g}-\mathrm{C}_{3} \mathrm{~N}_{4}$ photocathode for efficient photoelectrochemical water splitting. Appl. Catal. B: Environ. 2019, 118180.

S3. Liu, C.; Wang, F.; Zhang, J.; Wang, K.; Qiu, Y.; Liang, Q.; Chen, Z. Efficient photoelectrochemical water splitting by $\mathrm{gC}_{3} \mathrm{~N}_{4} / \mathrm{TiO}_{2}$ nanotube array heterostructures. NanoMicro Lett., 2018, 10, 1-37.

S4. Liu, C.; Qiu, Y.; Zhang, J.; Liang, Q.; Mitsuzaki, N.; Chen, Z. Construction of CdS quantum dots modified $\mathrm{g}-\mathrm{C}_{3} \mathrm{~N}_{4} / \mathrm{ZnO}$ heterostructured photoanode for efficient photoelectrochemical water splitting. J. Photochem. Photobiol. A 2019, 371, 109-117.

S5. Hajduk, Š.; Berglund, S.P.; Podlogar, M.; Dražić, G.; Abdi, F. F.; Orel, Z.C.; Shalom, M., Conformal Carbon Nitride Coating as an Efficient Hole Extraction Layer for ZnO Nanowires-Based Photoelectrochemical Cells. Adv.Mater. Interfaces, 2017, 4, 1700924.

S6. Fang, Y.; Xu, Y.; Li, X.; Ma, Y.; Wang, X. Coating polymeric carbon nitride photoanodes on conductive $\mathrm{Y}: \mathrm{ZnO}$ nanorod arrays for overall water splitting. Angew. Chem. 2018, 130, 9897-9901.

S7. Sun, M.; Fang, Y.; Kong, Y.; Sun, S.; Yu, Z.; Umar, A.; Graphitic carbon nitride (g$\mathrm{C}_{3} \mathrm{~N}_{4}$ ) coated titanium oxide nanotube arrays with enhanced photo-electrochemical performance. Dalton Trans. 2016, 45, 12702. 